University of Nebraska - Lincoln

DigitalCommons@University of Nebraska - Lincoln

Faculty Publications, Department of Psychology

Psychology, Department of

2009

Social Interaction Effects on Reward and Cognitive Abilities in

Monkeys

Jeffrey R. Stevens

University of Nebraska-Lincoln, jstevens5@unl.edu

Marc D. Hauser

Harvard University

Follow this and additional works at: https://digitalcommons.unl.edu/psychfacpub

Part of the Psychiatry and Psychology Commons

Stevens, Jeffrey R. and Hauser, Marc D., "Social Interaction Effects on Reward and Cognitive Abilities in Monkeys" (2009). Faculty Publications, Department of Psychology. 532.

https://digitalcommons.unl.edu/psychfacpub/532

This Article is brought to you for free and open access by the Psychology, Department of at DigitalCommons@University of Nebraska - Lincoln. It has been accepted for inclusion in Faculty Publications, Department of Psychology by an authorized administrator of DigitalCommons@University of Nebraska - Lincoln. 


\title{
Social Interaction Effects on Reward and Cognitive Abilities in Monkeys
}

\author{
Jeffrey R. Stevens and Marc D. Hauser \\ Department of Psychology, Harvard University, Cambridge, MA, USA
}

\section{Introduction}

When given a choice between receiving $\$ 100$ in 30 days and $\$ 110$ in 31 days, most people wait for the larger, more delayed reward. However, when the choice is between $\$ 100$ today and \$110 tomorrow, many people shift their preferences to the smaller, immediate reward despite the same difference of $\$ 10$ and one day. Reward properties - such as time to receipt-play a crucial role in our processing of rewards. However, reward processing does not only occur for individual decisions but also for social interactions; we must decide to either cooperate or compete with others for rewards. $\mathrm{Hu}-$ mans stand out in the animal kingdom as exceptional cooperators, both in terms of the form that cooperation assumes as well as the nature of rewards attained. Regarding form, we are unique in the stability of our reciprocal interactions and in the scale of our cooperative coalitions, entailing multiple nation states in times of war. In terms of rewards, we are of course motivated, like all other animals, by the central survival payoffs such as food and water but also by abstract entities such as money, the promise of future support, and positions of power such as a king, president, or head of an academic department. To maintain these complicated interactions and evaluate the nature of reward, we must possess a number of prerequisite cognitive abilities. Here, we view this problem through the lens of evolutionary biology, asking which aspects of our cognitive machinery, and the social interactions it supports, are uniquely human and which are shared with other primates. Though we focus on lemurs, monkeys, and apes, we acknowledge that many of the processes we document are unlikely to be restricted to the primates, and in many cases, there is already comparable evidence from other mammals and birds. We begin by reviewing a suite of cognitive mechanisms that are involved in both human and nonhuman primate reward processing. Our review is particularly focused on the subset of situations with quantifiable rewards. We then describe the kinds of social interactions that are part and parcel of primate life, especially the highly social monkeys and apes. Lastly, we merge these two sections and consider how constraints on primate cognition may limit the complexity of primate social interactions.

\section{Primate cognition}

To set up the problem of reward processing in animals, we can distinguish between asocial, individually based processes and social ones. The primary difference is whether access to rewards entails competitive or cooperative social interactions or instead can be achieved by an individual on its own. Some processes operate across asocial and social contexts. For example, an individual seeking food as a reward must discriminate between food and nonfood objects, quantify the amount of food available and its relative value, recall where food is located in the environment as well as the timing of its seasonal availability, and inhibit the temptation to take a small, immediately available reward over a larger, more valuable but delayed reward. Social interactions add a layer of complexity to these processes, raising questions about the extent to which individuals can generate expectations about what others want and intend, their competitive abilities, and the value of a coalitionary partner.

Primates are an ideal group in which to study the comparative cognition of reward processing because they are phylogenetically closely related to humans and because primate species vary in their life histories, mating systems, and foraging ecologies. Thus, we can not only explore how these different socioecological parameters uniquely shape each species' capacity for reward processing but also explore the extent to which there are more general, derived mechanisms, having evolved before humans diverged from their phylogenetic ancestors.

\section{Properties of rewards}

For folivorous, frugivorous, and omnivorous primates, individuals must distinguish between edible and inedible items, especially given the possibility of ingesting potentially toxic items. For many animals, recognition of edible foods entails using chemical cues, color patterns, textures, and shapes. And in some cases, these cues or features are detected in the context of social interactions. For example, in a study of rhesus macaques (Macaca mulatta), an experimenter ate one of two novel food items while a subject watched. The experimenter then placed new copies of each these food items on the ground and allowed the subject to approach and select one. Consistently, subjects picked the food item eaten by the experimenter. To assess which features mediated response selection, a set of follow-up conditions put different features into competition, using artificially created foods. For example, in one condition, the experimenter ate an orange sphere while holding a blue cube and then presented a blue sphere and an orange cube. Subjects consistently picked the orange cube, showing that in the context of a social foraging problem, rhesus macaques use color over shape to identify valuable food.

\section{Number}

To reliably process rewards, animals must be able to quantify reward amounts, either using number or some continuous dimension of amount such as volume. There are at least two naturally occurring situations in which number would appear to matter for primates: aggressive competition between groups and foraging for food. In black howler monkeys ( $\mathrm{Al}$ - 
ouatta pigra) and chimpanzees (Pan troglodytes), individuals attend to the number of competitors. Playback experiments demonstrate that a group of males is more likely to approach foreign males if the numerical odds are in their favor. Additionally, in many primate species, two or three individuals will form coalitions to defeat either a single dominant individual or a smaller coalition. Although these coalitions involve small numbers, they nonetheless require some capacity to quantify the number of competitors to acquire rewards such as mates or foraging areas.

Studies of foraging in animals show that individuals often maximize the rate of energetic returns, choosing patches with more over less food. Since estimates of rates of return depend on quantifying amount of food consumed over time, researchers have asked whether animals count the pieces, estimate the volume, or time the foraging periods in a patch. For instance, when given the choice between two different amounts of apple slices, rhesus macaques consistently picked two apples over one, three apples over two and four apples over three. But when presented with five versus four apple pieces, some animals picked four and some five. Without training, rhesus monkeys can quantify the number of pieces of food up to about four but thereafter appear to rely on a general estimate of amount.

Based on an overwhelming number of carefully controlled experiments, it is now fair to say that animals have a number sense that likely consists of two naturally available systems. One system allows animals to quantify individuals up to about four with precision; the second allows them to approximate number but without any limits on magnitude. In the precise system, as evidenced by the rhesus macaque example, primates are limited to representing four items simultaneously in short-term memory. Human infants and adults demonstrate a similar limit in a variety of tasks, suggesting continuity across development and across species. In the approximate system, primates can discriminate between approximate quantities using analog magnitudes, in which performance is limited by the ratio between the quantities and is independent of absolute value. Other work on operantly trained rhesus macaques demonstrates that ratio determines numerical discrimination between quantities ranging from 1 to 30 items. Ratio is important because it suggests that primate numerical competence conforms to Weber's law, a psychophysical process in which the accuracy of discriminating different quantities scales with the magnitude of the quantities. Again, human infants and adults also represent large approximate numbers and show similar signature ratio limits.

\section{Timing}

Predicting the timing of reward availability is a critical capacity for primates. In many species, crucial elements of the diet are only seasonally available, requiring both timing and planning. For example, when chimpanzees discover a source of ripe palm nuts, they not only invest in the processing of these nuts with stone hammers and anvils, but often leave behind particularly good hammers, retrieving them on future visits. Studies conducted with captive apes reveal that they can recall the kinds of tools needed for certain tasks, and retrieve them in the service of planning for future tasks. Based on their patterns of movement, it is also clear that many primates store information about fruiting cycles, enabling them to time the optimum period of visitation and harvesting.

Like number, the capacity to time events is based on a mechanism that, both behaviorally and neurobiologically, shows the signature of the analog magnitude system that underlies number. Importantly, timing exhibits the scalar property of Weber's law: the variance of timing accuracy scales with interval magnitude and is therefore a function of the ratio between magnitudes. For instance, rhesus macaques showed similar accuracy levels between timing events with a 1:1.5 ratio, regardless of whether the discrimination was $4 \mathrm{~s}$ vs. $6 \mathrm{~s}, 6 \mathrm{~s}$ vs. $9 \mathrm{~s}, 8 \mathrm{~s}$ vs. $12 \mathrm{~s}$, or $10 \mathrm{~s}$ vs. $15 \mathrm{~s}$. Unfortunately, little work has explored primate timing abilities beyond a few seconds, with the exception of periodic timing of circadian cycles. Moreover, the work is largely restricted to studies involving operant training, with no work on the relationship between timing and the role of social or ecological contexts.

\section{Impulsivity}

Animals must often combine quantity and temporal information to choose between rewards available over different time intervals (intertemporal choice). For example, imagine a monkey encounters a tree full of unripe fruit and with only a few ripe fruit: should it spend the time and energy to consume the few ripe fruit now or wait for the rest to ripen? Waiting would yield a higher reward amount, but the future is uncertain-another monkey may eat the fruit in the meantime, winds may knock them into a stream below, a fungus may infest and spoil the perfectly good fruit. This uncertainty may have provided a strong adaptive benefit for impulsivity.

Decision makers can make intertemporal choices by means of at least two mechanisms. The first mechanism simply assumes that the forager maximizes its food intake rate over repeated foraging bouts. This rate maximization account predicts that foragers should maximize the total energy gained from rewards over the total time spent acquiring and processing the rewards. Evidence from operant experiments suggests that cotton-top tamarins (Saguinus oedipus) and common marmosets (Callithrix jacchus) may use rate maximization strategies when trading off future benefits. Temporal discounting represents the second mechanism and involves the subjective devaluation of future rewards. If animals discount the future (as the example in the introduction implied), delayed rewards are less valuable than immediate rewards, potentially resulting in impulsive choice. In experiments that estimate discounting levels, subjects are presented with two stimuli: one associated with a small, immediate reward and the other with a large, delayed reward. The discounting level is "titrated" by incrementally increasing the delay-to-large until subjects are indifferent between choosing the large delayed reward and the small, immediate reward. Therefore, researchers can find indifference points between immediate and delayed rewards over a range of small and large reward amounts. Psychologists have exten- 
sively studied discounting in rats and pigeons, but fewer studies have investigated primate discounting. Surprisingly, the handful of primate discounting studies that have been conducted demonstrate that cotton-top tamarins, common marmosets, brown capuchin monkeys (Cebus apella), and rhesus macaques show similar discounting levels to rats and pigeons, devaluing rewards up to $50 \%$ in the first four seconds of delay!

The high levels of discounting observed for most nonhuman animals suggests that when rewards are distributed over time, individuals place a premium on immediacy, forgoing delayed rewards. This not only puts severe constraints on the foraging strategies of monkeys and apes, but constrains other behaviors such as cooperation, territory defense, and mate selection.

\section{Inhibitory control}

Inhibiting prepotent responses to reward is a difficult task for nonhuman animals. Clearly, natural selection has favored a strong drive to obtain the largest reward available. This is evidenced by the performance of a number of primate species on the reversed-contingency task in which subjects must reach toward the smaller of two visible rewards to receive the larger reward. Brown and black lemurs (Eulemur fulvus and E. macaco), tamarins, squirrel monkeys (Saimiri sciureus), rhesus macaques, orangutans (Pongo pygmaeus), gorillas (Gorilla gorilla), bonobos, and chimpanzees all invariably choose the large reward, only to receive the small rewardthey seem incapable of controlling their desire to reach for the larger reward amount.

The reversed-contingency task has proven difficult for primates to solve but not impossible. A number of techniques can circumvent the inhibitory control problem. First, sheer repetition can allow subjects to choose the smaller and receive the larger food reward. Most subjects do not solve the task after 200 trials, though some are at chance rather than repeatedly choosing the smaller amount. After 400-2500 trials, however, six rhesus macaques received the larger reward in over $90 \%$ of trials. Thus, massive exposure to the reversed contingencies can eventually trump the inhibitory problems. Second, imposing costs for "incorrect" choice can facilitate receiving the larger reward. By withholding all food when subjects pick the larger quantity, macaques and squirrel monkeys eventually learn to pick the smaller quantity; tamarins stick with the losing strategy, picking the larger and getting nothing at all. This suggests that part of the failure to point to the smaller quantity is due to the lack of costs associated with pointing to the larger quantity. Finally, including a mediator between seeing the food and receiving the food can curb the impulse to choose the larger reward amount. This has been most noticeably documented by an experiment testing chimpanzees trained on the Arabic number symbols from 1-6. Instead of choosing between one food treat versus four treats, the chimpanzees chose between a card with the numeral 1 written on its face and a card with the numeral 4; each card covered up the corresponding number of food treats. The chimpanzees quickly learned to pick the number 1 card and received 4 treats, indicating chimpanzees can learn a rule like "Point to the one you don't want to get the one you want". Surprisingly, once they learned this rule with symbols, they were incapable of generalizing to the same problem with food presented on its own. Therefore, it seems as though the difficulty of this task results from the chimpanzees' strong motivation to reach for food rather than an inability to learn the reverse-contingency rule.

\section{Memory}

Memory is a multi-faceted cognitive ability with clear evolutionary importance. Researchers have delineated several different types of memory that are applied to different types of information. Here we will focus on three types of memory: short-term, spatial, and episodic memory. One of the simplest tasks used to test short-term memory is the delayed-response task. In this task, a reward is placed under one of two (or more) identical but spatially separated stimuli. After a retention interval, subjects are allowed to chose one of the stimuli and receive the rewards if correct. Comparative data show that rhesus macaques are fairly good at remembering the location of the food item. They begin with about $80 \%$ accuracy after only a few seconds and drop to about $68 \%$ accuracy after a 15 second retention interval. Marmosets, however, though also starting at $80 \%$ accuracy, drop to $58 \%$ accuracy at 15 seconds, only slightly better than chance. Though some evidence suggests that the Old World monkeys may retain information better than the New World monkeys, this is by no means a universal, with capuchin monkeys being a notable exception to the rule.

Spatial memory plays an important role in primate reward acquisition. Recalling when and where food rewards are distributed is critical in foraging situations. In fact, some have suggested that foraging problems have posed strong evolutionary pressures on primate cognition, with species that feed on spatially and temporally variable foods showing higher levels of brain encephalization than species that feed on more stable food sources. Numerous studies have demonstrated strong spatial skills in primates, particularly in analogs to the radial arm maze. In this task, feeder stations placed throughout an enclosure are baited before subjects enter. After a subject finds all of the stations, researchers bait only some of the stations and, following a retention interval, record whether the subjects first attempt to feed at the previously baited stations. Most of the experiments on primates show high accuracy over retention intervals as long as 24 hours. As predicted by the adaptive specialization hypothesis, species that search over longer distances for food in the wild tend to be more accurate at longer retention intervals (e.g., frugivorous golden lion tamarins [Leontopithecus rosalia] are more accurate than gummivorous Wied's marmosets [Callithrix kulhi]). Additionally, the contingency between space and reward plays an important role. Common marmosets, for instance, are more accurate in a win-stay paradigm in which previous food locations predict current locations than in a win-shift paradigm in which subjects are not rewarded for visiting previously visited food stations. 
Probably one of the most interesting and controversial areas of primate memory is episodic memory. There is a feisty debate in the literature about whether nonhuman animals have the ability to mentally travel in time to recall previous experiences. The benchmark requirements for demonstrating episodic memory include showing that animals know "what," "where," and "when" an experience occurred. Though this is difficult to study in nonlinguistic creatures, recent research provides suggestive evidence of episodiclike memory in corvids and primates. For example, up to 24 hours after receiving a particular food item from an experimenter, a gorilla correctly identified the food type received and the experimenter from which he received it.

\section{Theory of mind}

Another highly contentious area of primate cognition is theory of mind. As mentioned earlier, this aspect of primate cognition is not strictly about reward. Yet, the capacity to make inferences about what others know, desire, believe and intend often leads directly to cooperative and competitive rewards, and most of the experimental studies use food as motivator to explore these mental states.

Early studies failed to find evidence of theory of mind in primates. One explanation for these failures was that the studies relied on animals making inferences about human mental states in contexts involving cooperation, as opposed to asking animals to make inferences about each other in the context of competition. Though several primate species do cooperate (see below), competition is far more common and may represent the most basic form of social interaction among animals. To test this hypothesis, recent studies of monkeys and apes have used both competitive and cooperative tasks, contrasting conspecific pairing as well as conspecific-heterospecific pairings. There is increasing evidence that monkeys and apes make inferences from seeing to knowing. Chimpanzees, for instance, can take the visual perspective of other individuals to understand whether they can see occluded food rewards: subordinate chimpanzees preferentially approach rewards that are obscured from the view of dominant individuals. Rhesus monkeys show similar abilities, stealing food primarily from experimenters without visual access to the food rather than from those that do have access. Though visual perspective taking is only one component of having a theory of mind, it plays a significant role in the competitive and cooperative interactions of primates. What remains to be explored is whether primates go beyond this elemental capacity, attributing intentions, desires, and beliefs to others.

\section{Primate social interactions}

In socially living animals, such as some of the lemurs and most monkeys and apes, obtaining rewards is often coupled with socially competitive or cooperative interactions. In some instances, helping another individual yields payoffs for the helper, while in other cases, helping is costly for the helper. The existence of the latter poses a puzzle given the generally accepted Darwinian assumption that behavior evolves via natural selection to provide fitness benefits to the individual. Specifically, what selective pressure has favored individuals who provide benefits to other individuals?

Here, we address the evolution of cooperation by describing four different models (mutualism, kin selection, reciprocity, and punishment), focusing on evidence from monkeys and apes. We operationally define cooperation as social interactions resulting in a net benefit for all participants; here, benefit is measured in terms of increasing reproductive success.

\section{Mutualism}

The simplest type of cooperative behavior provides direct benefits to the cooperator, in addition to other individuals. Therefore, benefits to others are by-products of benefits to self. This model of cooperative behavior is termed by-product mutualism. Any individual that defects (does not cooperate) in mutualistic situations will, by definition, do worse than a cooperator; therefore, in the absence of a temptation to defect, cooperation provides the best option. Importantly, mutualism does not depend on the identity of one's partner and thus can occur between any members of the same species and even members of different species.

Mutualism is a common form of cooperation, with cooperative hunting providing a prime example. When multiple individuals cooperate to hunt the same prey, they can both increase the probability of successful prey capture and reduce the individual costs associated with hunting. Cooperative hunting provides mutualistic benefits only when the per capita intake rate increases with group size. Therefore, a pair of hunters would have to capture more than twice as many prey items as a solitary hunter. This typically requires the success rate of solitary hunters to be fairly low, making cooperation particularly successful. Increased success combined with lower hunting costs can lead to direct, immediate, and simultaneous fitness benefits for cooperative hunters, particularly when hunting small or difficult prey. For example, wild chimpanzees often cooperatively hunt arboreal monkeys. Although different populations vary, there is evidence that in some populations the per capita intake rate increases with group size, suggesting that the hunting is cooperative. Cheaters that do not hunt are discouraged by receiving only small amounts of meat in food sharing bouts following a kill. Thus, it is in an individual's best interest to participate in a hunt to acquire the much sought-after meat.

In the laboratory, mutualism has been documented in a variety of primates including marmosets, capuchin monkeys, and chimpanzees. Typically, when joint action is required to receive separate benefits for each player, subjects succeed in cooperating. However, when food is monopolizable instead of divided between players, cooperation breaks down. That is, when immediate rewards are available, primates cooperate. But once competition reduces the probability of reward, there is no guarantee of immediate benefit and no apparent mechanism capable of maintaining cooperation. In a recent study of captive chimpanzees, individuals 
confronted an opportunity to manipulate an apparatus for a food reward; on some trials, the focal subject had to recruit the help of another individual in order to manipulate the apparatus and on other trials, the focal could operate the apparatus alone. Subjects only recruited help when two individuals were required to obtain the food reward. Moreover, in a second experiment, subjects selectively recruited effective helpers over ineffective helpers, demonstrating a keen knowledge of the role of others in the mutualistic acquisition of rewards.

\section{Kin selection}

Whereas mutualism poses no problem for classic Darwinian theory, altruistic cooperation does as the actor incurs a cost while benefiting another. Kin selection provided the first clear theoretical solution to the paradox of altruism. Individuals may bias cooperation toward their genetic relatives because it helps propagate their own genes shared by common descent. What looks altruistic from an individual's perspective actually serves self-interest from the gene's view. Individuals have a certain probability of sharing a gene $(r$-the coefficient of relatedness) with relatives due to common descent. If the benefits to kin discounted by this coefficient of relatedness outweigh the costs of helping, altruism towards kin can evolve.

Kin selection may be particularly powerful in primates because of the close-knit family groups found in many species. With a few notable exceptions, including chimpanzees, nonhuman primates follow the general mammalian pattern of philopatry in which males leave their natal groups upon reaching reproductive maturity whereas females stay in their natal groups for life; consequently, most primate groups consist of closely related female kin and genetically unrelated males. Kin selection theory predicts that individuals should preferentially help relatives and should help in proportion to their coefficient of relatedness. These types of quantitative predictions have proven difficult to test in primates, but there are data showing kin-directed helping, particularly in coalition formation and food sharing.

Primates frequently form coalitions in which multiple individuals form an alliance in agonistic interactions with other group members. This is particularly prevalent in primate species with matrilineal dominance hierarchies, such as macaques and baboons. In these species, females often assist relatives in aggressive encounters with other group members. Notably, the probability of coalitionary support tapers off with increasingly distantly related kin: mothers almost always aid offspring but are less likely to aid nieces and nephews. Therefore, as relatedness increases, these primates are more likely to pay costs associated with helping kin. Additionally, individuals are more likely to form coalitions with kin than nonkin against higher-ranking opponents, suggesting that they will pay a higher cost of helping related individuals. Male primates can also form coalitions, often to guard or acquire sexually receptive females. In situations in which few mating opportunities exist for males, these kin-based coalitions are especially relevant because aiding kin helps ensure the propagation of one's genes, even in the absence of direct mating opportunities. This is the case in red howler monkeys (Alouatta seniculus) where coalitions of related individuals have longer tenure in a troop (and therefore more mating opportunities) than unrelated individuals. Though many instances of coalitionary support are directed to kin, some are directed to nonkin and may be attributable to other benefits such as mutualism and reciprocity.

One of the most often-reported examples of apparent altruism is food sharing-voluntarily allowing another to consume food that one individual possesses. Food sharing is, of course, very common between mother and offspring for numerous primate species. However, it also occurs between grandparents and grandoffspring, siblings, and even adult relatives, often scaling with relatedness. In captive macaques, for instance, mother-daughter pairs $(r=0.5)$ frequently fed from the same food bin. Both grandmothergranddaughter pairs and sister-sister pairs $(r=0.25)$ co-fed at intermediate rates, and aunt-niece pairs $(r=0.125)$ co-fed at low levels indistinguishable from unrelated pairs. Though it is unclear what proportion of food transfers occur between relatives, kin-based sharing likely explains the bulk of sharing in numerous species.

\section{Reciprocity}

Reciprocity occurs when an individual pays a short-term cost of cooperation for the future benefit of a social partner's reciprocated cooperation. As a form of altruistic cooperation, reciprocity aims to explain cooperative behavior among genetically unrelated individuals (thereby eliminating kin selection as an explanation) in a unique type of social interaction termed the prisoner's dilemma. The keys aspects of the prisoner's dilemma are: 1) cooperation maximizes the total payoff to everyone involved in the interaction (mutual cooperation provides more benefits than mutual defection); however, 2) any individual will receive a higher personal payoff by defecting, so a sizable temptation to cheat exists. Pursuing unilateral cooperation in this game is not a stable strategy.

Theoreticians suggested that reversing roles as donor and recipient of altruism may reduce the temptation to defect because it largely commits individuals to invest in future cooperation. When the fitness payoffs sum over a series of interactions with the same partner, reciprocal strategists can reap the benefits of mutual cooperation. The reciprocal strategy tit-for- tat, in which a player starts out cooperating and copies its opponent's behavior in previous interactions, can successfully invade and dominate simulated populations of social partners engaging in prisoner's dilemma games, winning out over many alternative behavioral strategies. If the probability of interacting again exceeds a critical level, a reciprocal strategy can maintain cooperation.

Many authors have reported examples of reciprocity in animals, including primates, spanning such contexts as food sharing, grooming, and coalitions associated with mating opportunities. Unfortunately, in most cases these pur- 
ported examples of reciprocity have either never been replicated or have been challenged by alternative explanations for the patterns of interaction, including especially kin selection and mutualism. Another alternative explanation, symmetry-based reciprocity, occurs when the reciprocal pattern can be explained by the symmetrical relationship between individuals. For instance, if individuals A and B interact frequently and cooperate, their behavior will appear to be reciprocal even though it is based on symmetrical interactions rather than contingent cooperation. This contrasts with calculated reciprocity (such as tit-for-tat) in which individuals track debts owed and favors given when deciding whether to cooperate.

A field study of olive baboons (Papio anubis) provided one of the first reports of reciprocity. Males formed coalitions in order to drive off rival males and gain access to reproductively active females. Data suggested that males took turns reaping the benefits, implying evidence of reciprocity. The robustness of this finding, however, is unclear given that a subsequent study of a different population of baboons did not find the same reciprocal patterns and a study of a closely related species proposed an alternative, mutualistic explanation.

Another example comes from experimental tests of food sharing in captive capuchin monkeys. When pairs of capuchins were placed in adjacent chambers separated by a mesh partition, if individual A allowed individual B to consume dropped scraps of food in one particular trial, then B tended to allow A to gather scraps in the next trial. Because the food transfer is primarily passive and costs may be minimal, these results can be explained by a mutual tolerance between individuals. Therefore, the capuchins do not appear to be assessing the costs and benefits of their actions, making calculated reciprocity an unlikely explanation of capuchin food transfers.

Lastly, in a series of experiments with captive cottontop tamarins, subjects could pull a tool to give food to an unrelated recipient without getting any food for self. Tamarins pulled the tool most often for partners that always pulled and infrequently for partners that never pulled. The frequency of cooperation was, however, less than $50 \%$, and as each game progressed, the amount of food given dropped. Tamarins, therefore, maintained moderate levels of cooperation with other cooperators, but the degradation of cooperation over time suggests an unstable system; in fact, game theoretic modeling of the data suggest that reciprocity crashes if an opponent fails to cooperate on two consecutive opportunities.

In summary, there are few examples of true, calculated reciprocity among monkeys and apes. Most putative examples can be explained by symmetry-based reciprocity, mutualism, or kin selection. Given the simplicity of the preconditions for reciprocity and the apparent ubiquity of reciprocity in humans, it's rarity in primates (and other animals) is surprising. We take up this issue in the section on cognitive constraints on reward processing.

\section{Punishment}

Punishing individuals that defect can potentially impose enough costs to offset the temptation to cheat and, like reciprocity, can elicit future cooperation. Punishment involves energetic costs and, when accomplished by means of aggression, also involves the costs of risked injury. Consequently, as an adaptive behavior, punishment is a selfish strategy, favoring actions that ultimately benefit the punisher by eliciting cooperative behavior from the recipient of punishment. As defined, punishment resembles reciprocity, which should occur when it elicits cooperative behavior directed strictly at the reciprocator. Despite the theoretical interest, punishment is not well-documented in animals; in fact, though animals act aggressively toward others in the context of resource defense (e.g., territoriality, dominance interactions), there are few examples in the context of cooperation.

Whereas punishment penalizes past behavior with the hope of future reward, a similar behavior called harassment penalizes present behavior to elicit immediate reward. Harassment occurs when an individual imposes costs such as aggression on another to induce immediate cooperation, thereby providing instant benefits to the harasser. Harassment may also influence future as well as current cooperation, suggesting that it can lead to punishment strategies. For instance, if a defector is in the process of consuming an entire food resource, a harasser could impose costs on the defector aimed at obtaining some portion of the food resource immediately. Whereas punishment appears relatively rare among animals, harassment may be more common. For instance, after capturing prey, chimpanzees frequently allow other individuals to consume part of the meat. Observational work has demonstrated that harassment best accounts for the pattern of food sharing, taking the form of individuals incessantly begging for food with hand gestures, grabbing at the food, and placing their hands over the mouths of the food possessors. In this context, harassment was costly for the food owner (food intake rate decreased as the number of beggars increased), owners shared more often when beggars harassed frequently and intensely, and when sharing occurred harassment levels decreased. Controlled experiments corroborated these findings with captive chimpanzees, as well as squirrel monkeys (Saimiri boliviensis), a species that rarely cooperates.

Harassment may, of course, influence future as well as current cooperation, suggesting that it may lead to punishment strategies. One example comes from rhesus macaques. When individuals find food, they often give food calls, an altruistic act that recruits others to the food source, thereby potentially benefiting kin. However, individuals sometimes find food and suppress their calls. If no one detects these silent discoverers, they reap more food rewards than those who announce their discoveries. If, in contrast, they are detected, then they are aggressively attacked, leading to an even larger loss of food to the attacker. Thus, aggression yields an immediate benefit of accessing food for the attacker, clearly qualifying it as a case of harassment. In addition, the aggression may have a punishing effect on silence by eliciting future food calls. That is, aggressive behavior influences both immediate and future benefits. 


\section{The cognitive psychology of a cooperator}

Many researchers have argued that the frequency of complex social interactions in primates underlies the evolution of their cognitive abilities. Negotiating a complicated network of allies and enemies, dominants and subordinates, and mates and competitors has created selective pressure for cognitive abilities such as transitive inference, theory of mind, and deception. Though this connection between sociality and cognition has been well recognized, the cognitive constraints on social interaction have often been ignored. Put simply, much of the work on primate cognition has focused on how large brains enable rich social interactions instead of how limited cognitive abilities may constrain the range of social interactions. Here, we take up this "cognitive constraints" perspective.

Mutualism and kin selection are both theoretically well-understood and empirically well-documented models of cooperation. By contrast, reciprocity and punishment, while theoretically feasible, are rare among primates (although harassment may be more widespread). Therefore, despite models purporting the evolutionary stability of all of these types of cooperation, some types occur much more frequently than others. Unfortunately, a strictly adaptive perspective has limited power to explain the frequency of mutualism and kin-biased cooperation and the rarity of reciprocity and punishment. A proximate perspective that links with the ultimate problem can, however, reveal how cognitive constraints limit or facilitate particular forms of cooperation.

The proximate approach emphasizes critical aspects of reciprocity and punishment that differ markedly from mutualism and kin-biased cooperation. In both reciprocity and punishment, the fitness benefits associated with cooperation depend on the partner's behavior: cooperation should only occur when the partner responds by reciprocating or punishing. When this contingent response occurs in the future, the temporal delay introduces cognitive challenges that may constrain the emergence and stability of cooperation. Animals can easily implement strategies that yield immediate benefits, such as mutualism and harassment, because individuals do not have to track benefits over time. With a time delay between cooperating and receiving return benefits, however, individuals must invest in an uncertain future. Delayed benefits impede learning the consequences of cooperation, place greater demands on the capacity to recall previous interactions, and trade off short-term fitness gains for long-term gains. Here we highlight how several key cognitive constraints play a role in mutualism, kin-biased cooperation, reciprocity, and punishment, thereby uniting the two sections of this essay.

\section{Cognitive constraints on mutualism and kin-biased cooperation}

Because no temptation to cheat exists in mutualistic interactions, individuals should always cooperate. As a result, mutualism requires no special cognitive abilities above and beyond the challenges inherent in the cooperative behavior itself. Kin-biased cooperation does, however, require additional cognitive capacities. At a minimum, it requires the capacity to direct cooperative actions to related individuals.

Mechanisms of kin recognition include recognition alleles, phenotype matching, and spatial and familiarity cues. The recognition allele hypothesis predicts that individuals can compare a particular phenotypic cue (auditory, olfactory, visual, etc.) to an innately specified template. Such a model requires few cognitive skills other than discriminating the cue associated with relatedness. The closely related phenomenon of phenotypic matching occurs when an individual compares a conspecific's phenotypic cues to a learned template. This requires specialized perceptual and computational systems that detect cues at an early stage to form a template, then test cues against the template to discriminate kin. Though recognition alleles and phenotypic matching provide the most direct forms of kin recognition and have been observed in some animal species, they do not appear to be common mechanisms of primate kin recognition. Instead, primates seem to use a simple set of rules such as spatial and familiarity cues to discriminate kin. Often primates may use rules such as "be nice to individuals near your home" or "help those that you grew up with" to direct the benefits of cooperation towards kin. These familiarity mechanisms require little in the way of cognitive abilities, building on general laws of learning by association, and seem to be widespread throughout the primates.

\section{Cognitive constraints on reciprocity and punishment}

The original formulation of reciprocity had three requirements for evolutionary stability: 1) the reciprocated benefit must outweigh the immediate cost, 2) individuals must interact repeatedly, and 3) individuals must recognize each other. These requirements, however, most likely underestimate what is cognitively necessary for both developing and maintaining a system of stable reciprocity. In particular, the delay between the cost of a cooperative act and the benefit of reciprocated cooperation introduces a number of cognitive challenges. Like reciprocity, punishment can involve a delay between a costly act and a beneficial payoff, and in these cases it faces similar constraints. For this reason, we consider the constraints on reciprocity and punishment together, pointing to differences where appropriate.

Individual recognition - Like kin selection, reciprocity and punishment require directing cooperation to others. Unlike kin selection, however, where altruistic acts are disseminated simply as a function of coefficients of genetic relatedness, reciprocity and punishment require targeting specific individuals. Therefore, the delayed, contingent response re- 
quired for both reciprocity and punishment necessitates that individuals can distinguish different partners. Mechanisms for individual recognition appear to be the norm among animals, including lemurs, monkeys, and apes. Consequently, the paucity of evidence for reciprocity and punishment across primates cannot appeal to individual recognition as a constraint.

Number, amount and time-Numerical abilities can play a key role in reciprocity when individuals must precisely quantify the reward amounts being reciprocated. When primates engage in a bout of reciprocity, they will either be limited to small numbers of objects in cases where the exchange must be precise (a banana for a banana), or they will be freed from this constraint where approximate exchanges are tolerated. The same prediction holds for cases where the currency is time, such as the duration of a grooming bout. If one monkey grooms another for 10 minutes, the groomer will most likely accept as fair exchange a reciprocated grooming bout of between 8-12 minutes. As reward quantity and time magnitudes increase, quantification accuracy decreases, making equitable exchange more difficult and leaving opportunities for cheaters to exploit the judgment errors. This constraint only applies when variance exists in the quantity and time magnitudes. If the exchange is always a banana for a banana, these quantification abilities are superfluous.

Inhibitory control and impulsivity-Reciprocity is itself an inhibitory and impulsivity problem: can an individual inhibit the choice of the immediate large benefit to gain the delayed larger benefit? Like the reversed-contingency task, the chooser must first reach for the undesirable over the desirable reward. The first move can be likened to giving away food. By avoiding the temptation of the larger immediate reward, the first move is costly to self. As data on the reversedcontingency task in primates show, the cost often appears too great and the inhibitory system too weak.

The second move, waiting for the reciprocated benefit, is analogous to the intertemporal choices described earlier. Individuals must choose between the immediate reward of defecting and the long-term reward of cooperating. Indeed, a number of researchers have predicted that impulsivity can reduce the value of reciprocated benefits. Experimental data on variation in human impulsivity and cooperation validate the view that a preference for immediate rewards may inhibit reciprocity. Impulsivity correlates with cooperation such that individuals who prefer immediate gains also cooperate less frequently. In parallel, blue jays (Cyanocitta cristata) show stable cooperation in a prisoner's dilemma only following an experimental reduction in their preference for immediacy. Although primates have not been tested in both impulsivity and cooperation tasks, it seems highly probable that a significant constraint on their capacity for reciprocity emerges from their steep discounting functions. Similar abilities are required for punishment: punishers must pay an immediate cost of punishing to achieve a future, discounted reward.
Memory - Episodic memory may very well be required to implement reciprocity. Because individuals must track the actions ("what") of previous partners ("who") the last time that they interacted ("when"), fairly sophisticated memory systems may be required for reciprocity. Even if episodic memory itself is not required, limitations in memory decay, interference, and capacity can also constrain the frequency of reciprocity and punishment. Memory decay proceeds rapidly over time; therefore, longer time intervals between cooperative acts may make reciprocity and punishment more difficult. Even with short time delays between cooperative interactions and few distractions, every potential new partner increases the computational load of tracking debts owed, favors given, and costs imposed. Keeping score of reciprocal obligations and punishment with the extensive social network found in many primate species may place a computationally intensive burden on memory systems. Although few studies examine learning and memory constraints in primate cooperation, studies of human cooperation suggest that these constraints can pose challenges for maintaining stable cooperative relationships.

Theory of mind - Understanding the knowledge of others is critical to strategic reasoning. "I know that you know that I know that you know..." is a central assumption of economic decision making required for rational agents to solve economic games. If, however, natural selection acts as the "rational agent" by selecting for individuals that make good decisions, this capacity may not be required for individuals if they use simple rules that approximate the outcome of fully rational choice. Therefore, it is possible for primates to implement reciprocity without full theory of mind capacities. At present, only a few studies have attempted in integrate issues concerning mental state attribution with those involved in cooperation. For example, recent demonstrations of visual perspective taking abilities in primates suggests that they might be able to attend to rewards that others seek, possibly aiding their ability to recognize cooperative situations. However, despite this evidence, recent work shows that chimpanzees do not demonstrate other-regarding preferences; that is, they do not preferentially acquire food rewards in such a way as to give food to others. Instead, they appear to be completely indifferent to how their own reward-based actions can benefit other individuals. In contrast, in the study of tamarins mentioned above, individuals were more likely to cooperate with individuals who intentionally gave food as an altruistic act as opposed to individuals who gave the same amount of food as an accidental by-product of an otherwise selfish act. Further study of what monkeys and apes understand of the goals, beliefs, and intentions of others will elucidate whether limitations in these areas constrain otherregarding preferences.

\section{Conclusions}

With increasingly sophisticated and ecologically relevant tasks, we are closing in on those aspects of reward process- 
ing and social cognition that have uniquely evolved in humans and those that evolved by common descent with other primates, and possibly, other nonprimate animals. We suggest reward processing and social cognition are intimately linked, setting up cognitive constraints on the evolution of cooperative behavior. We do not propose that these reward processing constraints are immutable. If the evolutionary benefit of cooperation is great, selection pressure may circumvent these constraints. This, however, is likely to occur in a domain-specific manner rather than a domain-general manner. For instance, food caching species such as squirrels may suspend inhibitory and impulsivity problems when hiding nuts away for winter. Yet, this ability has evolved to solve a very specific problem and likely does not apply generally to the species' decision-making strategies. Therefore, domain-general cognitive systems must evolve to allow many of the more complex forms of cooperation. The possibly unique presence of reciprocal cooperation and punishment in humans may be directly attributable to a combination of our keen number sense, patience, inhibitory control, memory capacity, and theory of mind.

\section{Further reading}

Axelrod, R., \& Hamilton, W. D. (1981). The evolution of cooperation. Science 211, 1390-1396.

Boesch, C., \& Boesch, H. (1989). Hunting behavior of wild chimpanzees in the Tai National Park. American Journal of Physical Anthropology 78, 547-573.

Boysen, S. T., \& Berntson, G. G. (1995). Responses to quantity: Perceptual versus cognitive mechanisms in chimpanzees (Pan troglodytes). Journal of Experimental Psychology: Animal Behavior Processes 21, 82-86.
Chapais, B., \& Berman, C. M. (2004). Kinship and behavior in primates. Oxford: Oxford University Press.

Hampton, R. R., \& Schwartz, B. L. (2004). Episodic memory in nonhumans: What, and where, is when? Current Opinion in Neurobiology 14, 192-197.

Hare, B., Call, J., Agnetta, B., \& Tomasello, M. (2000). Chimpanzees know what conspecifics do and do not see. Animal Behaviour 59, 771-785.

Hauser, M. D., Chen, M. K., Chen, F., \& Chuang, E. (2003). Give unto others: Genetically unrelated cotton-top tamarin monkeys preferentially give food to those who altruistically give food back. Proceedings of the Royal Society of London, Series B 270, 2363- 2370.

Hauser, M. D., \& Spelke, E. S. (2004). Evolutionary and developmental foundations of human knowledge: A case study of mathematics. In Gazzaniga, M. (ed.), The cognitive neurosciences III. pp 853-864. Cambridge, MA: MIT Press.

Kacelnik, A. (2003). The evolution of patience. In Loewenstein, G., Read, D. \& Baumeister, R. F. (eds.) Time and decision: Economic and psychological perspectives on intertemporal choice. pp 115-138. New York: Russell Sage Foundation.

Meck, W. H. (2003). Functional and neural mechanisms of interval timing. Boca Raton, Fla.: CRC Press.

Stevens, J. R. (2004). The selfish nature of generosity: Harassment and food sharing in primates. Proceedings of the Royal Society of London, Series B 271, 451-456.

Stevens, J. R., Hallinan, E. V., \& Hauser, M. D. (2005). The ecology and evolution of patience in two New World monkeys. Biology Letters 1, 223-226.

Tomasello, M., \& Call, J. (1997). Primate cognition. Oxford: Oxford University Press.

Trivers, R. L. (1971). The evolution of reciprocal altruism. Quarterly Review of Biology 46, 35-57. 